\title{
Impact on the Hipparcos2-UCAC4 geometric relation from some physical properties of the stars
}

\author{
Francisco J. Marco ${ }^{1}$, María J. Martínez ${ }^{2}$ and Jose A. López ${ }^{1}$ \\ ${ }^{1}$ Universidad Jaume I. Dept. Matemáticas, IMAC. \\ Avda. Vicent Sos Baynat s/n, 12071, Castellón, Spain \\ email: marco@mat.uji.es, lopez@mat.uji.es \\ ${ }^{2}$ Universidad Politécnica de Valencia. Dept. Matemática Aplicada. IUMPA. \\ Camino de Vera s/n. 46022 Valencia. Spain \\ email: mjmartin@mat.upv.es
}

\begin{abstract}
The aim of this paper is the study of the impact that the consideration of different physical properties as magnitude and spectral type of stars has on the geometric relations between Hipparcos 2 and UCAC4. In this sense, the pairs of residuals $\Delta \alpha^{*}$ and $\Delta \delta$ can be considered as functions of $(\alpha, \delta, r)$ and for each fixed $r$, we can fit a vector field on the sphere from which to obtain its components in the VSH basis. The same can be done by grouping the stars considering their magnitudes, spectral types (or mixing them) and then studying the variations in the mentioned geometry. We must not forget that $\Delta \alpha^{*}$ and $\Delta \delta$ are numerical random variables whose regression on the magnitude $m$, for example, can be estimated. The results will be computed taking into account $r$ as well as the physical mentioned properties. So, we avoid the assumption that the harmonic coefficients depend only on $m$.
\end{abstract}

Keywords. astronomical data bases: miscellaneous, catalogs, reference systems

\section{Magnitudes and distances}

We have selected two sets of common stars from Hipparcos and UCAC4 regarding their magnitudes. We call these sets m5 (23282 stars with magnitudes ranging [7.153, 8.556]) and $\mathrm{m} 6$ (31753 stars ranging [8.556, 9.959]), and distances up to 800 pcs. We study the different behaviors in $\Delta \alpha^{*}$ and $\Delta \delta$. Both magnitudes cover the $80 \%$ of the total population that has been considered (79591 non-double stars and with strictly positive parallaxes) If we proceed as in Marco et al. (2017), we see that around $r=400$ pcs, for both $\mathrm{m} 5$ and $\mathrm{m} 6$ populations, there are possible problems for the vector field of the residuals (possible saddle point for $\mathrm{m} 5$ and almost coincident, on a long range of $r$, for m6, which could imply a possible degeneration).

Table 1 shows that the behavior of the coefficients is different for both populations, as expected. Globally $s_{10}$ and $t_{1-1}$ decrease with $r$, being similar for the two consecutive distances 300 and 400pcs. We also find this behavior in $s_{11}$. On the other hand, $t_{10}$ decreases slightly. Finally, $s_{1-1}$ and $t_{11}$ have unparalleled behaviors. We also notice that formal errors are generally high, although a more detailed study shows that the stars collected in $\mathrm{m} 6$ have a more stable behavior.

\section{Spectral type and distances. Mixing properties}

The spectral type, by itself, does not provide relevant information, but this changes if we consider the data together with the J-magnitude. We used stars up to 800 pcs 
Table 1. Results for $\mathrm{m} 5, \mathrm{~m} 6$ and weighted $\mathrm{m} 5+\mathrm{m} 6$ sets

\begin{tabular}{|c|c|c|c|c|c|c|}
\hline$m_{5}(\mathbf{p c s})$ & $s_{1,0}$ & $s_{1,1}$ & $s_{1,-1}$ & $t_{1,0}$ & $t_{1,1}$ & $t_{1,-1}$ \\
\hline $\begin{array}{l}S^{2} \\
{[200,400]} \\
{[300,500]}\end{array}$ & $\begin{array}{l}9.68 \pm 0.02 \\
5.08 \pm 0.80 \\
3.28 \pm 0.14\end{array}$ & $\mid \begin{array}{l}2.42 \pm 0.02 \\
4.44 \pm 0.50 \\
4.04 \pm 0.25\end{array}$ & $\begin{array}{c}0.24 \pm 0.02 \\
0.16 \pm 0.01 \\
-1.43 \pm 0.35\end{array}$ & $\left|\begin{array}{l}4.16 \pm 0.02 \\
5.15 \pm 0.40 \\
5.63 \pm 0.30\end{array}\right|$ & $\begin{array}{c}0.08 \pm 0.02 \\
-1.09 \pm 0.40 \\
-1.32 \pm 0.11\end{array}$ & $\begin{array}{l}3.50 \pm 0.02 \\
5.56 \pm 0.12 \\
3.53 \pm 0.03\end{array}$ \\
\hline$m_{6}(\mathbf{p c s})$ & $s_{1,0}$ & $s_{1,1}$ & $s_{1,-1}$ & $t_{1,0}$ & $t_{1,1}$ & $t_{1,-1}$ \\
\hline $\begin{array}{l}S^{2} \\
{[200,400]} \\
{[300,500]}\end{array}$ & $\begin{array}{l}8.83 \pm 0.01 \\
6.36 \pm 0.31 \\
4.62 \pm 0.63\end{array}$ & $\begin{array}{l}2.88 \pm 0.01 \\
3.30 \pm 0.10 \\
2.90 \pm 0.01\end{array}$ & $\begin{array}{l}0.79 \pm 0.01 \\
1.48 \pm 0.43 \\
1.91 \pm 0.25\end{array}$ & $\left|\begin{array}{l}2.65 \pm 0.01 \\
2.11 \pm 0.01 \\
1.17 \pm 0.02\end{array}\right|$ & $\begin{array}{l}1.77 \pm 0.01 \\
0.44 \pm 0.38 \\
0.96 \pm 0.05\end{array}$ & $\begin{array}{l}3.44 \pm 0.01 \\
2.99 \pm 0.18 \\
1.77 \pm 0.35\end{array}$ \\
\hline$m_{5}+m_{6} \quad(\mathbf{p c s})$ & $s_{1,0}$ & $s_{1,1}$ & $s_{1,-1}$ & $\iota_{1,0}$ & $\iota_{1,1}$ & $\iota_{1,-1}$ \\
\hline $\begin{array}{l}S^{2} \\
{[200,400]} \\
{[300,500]}\end{array}$ & $\begin{array}{l}9.19 \pm 0.03 \\
5.81 \pm 0.40 \\
4.05 \pm 0.60\end{array}$ & $\mid \begin{array}{l}2.69 \pm 0.03 \\
2.10 \pm 0.02 \\
3.38 \pm 0.30\end{array}$ & $\begin{array}{l}0.56 \pm 0.03 \\
0.92 \pm 0.30 \\
0.50 \pm 0.07\end{array}$ & $\begin{array}{l}3.29 \pm 0.03 \\
5.86 \pm 0.30 \\
3.63 \pm 0.04\end{array}$ & $\begin{array}{l}1.06 \pm 0.03 \\
3.40 \pm 0.20 \\
0.00 \pm 0.30\end{array}$ & $\begin{array}{l}3.47 \pm 0.03 \\
4.08 \pm 0.10 \\
2.51 \pm 0.12\end{array}$ \\
\hline
\end{tabular}

Table 2. Results for $\mathrm{m} 5$ and $\mathrm{m} 6$ sets mixed with KM and No-KM stars

\begin{tabular}{|c|c|c|c|c|c|c|}
\hline$K M-m_{6}$ (pcs) & $s_{1,0}$ & $s_{1,1}$ & $s_{1,-1}$ & $t_{1,0}$ & $t_{1,1}$ & $t_{1,-1}$ \\
\hline \begin{tabular}{|l|}
$S^{2}$ \\
{$[200,400]$} \\
{$[300,500]$} \\
\end{tabular} & $\begin{array}{l}5.85 \pm 0.05 \\
4.16 \pm 0.80 \\
3.21 \pm 0.50\end{array}$ & $\left|\begin{array}{l}2.54 \pm 0.01 \\
2.99 \pm 0.20 \\
3.74 \pm 0.30\end{array}\right|$ & $\left|\begin{array}{c}-0.23 \pm 0.01 \\
0.34 \pm 0.30 \\
0.13 \pm 0.01\end{array}\right|$ & $\begin{array}{l}6.58 \pm 0.01 \\
5.36 \pm 0.12 \\
5.33 \pm 0.15\end{array}$ & $\begin{array}{l}2.99 \pm 0.02 \\
3.34 \pm 0.30 \\
3.06 \pm 0.04\end{array}$ & $\begin{array}{l}2.06 \pm 0 . \\
1.51 \pm 0 . \\
1.45 \pm 0 .\end{array}$ \\
\hline$\left|N o-K M-m_{6}(\mathbf{p c s})\right|$ & $s_{1,0}$ & $s_{1,1}$ & $s_{1,-1}$ & $t_{1,0}$ & $t_{1,1}$ & $t_{1,-1}$ \\
\hline \begin{tabular}{|l|}
$S^{2}$ \\
{$[200,400]$} \\
{$[300,500]$} \\
\end{tabular} & \begin{tabular}{|c|}
$11.43 \pm 0.02$ \\
$9.87 \pm 0.90$ \\
$10.43 \pm 0.04$
\end{tabular} & $\left|\begin{array}{l}3.70 \pm 0.10 \\
3.75 \pm 0.10 \\
1.85 \pm 0.10\end{array}\right|$ & $\begin{array}{l}1.56 \pm 0.02 \\
1.75 \pm 0.60 \\
2.96 \pm 0.16\end{array}$ & $\begin{array}{c}0.97 \pm 0.02 \\
-0.48 \pm 0.21 \\
-1.57 \pm 0.06\end{array}$ & $\begin{array}{c}1.23 \pm 0.02 \\
-1.00 \pm 0.73 \\
-2.72 \pm 0.60\end{array}$ & $\begin{array}{l}3.92 \pm 0.04 \\
4.08 \pm 0.05 \\
1.63 \pm 0.35\end{array}$ \\
\hline$K M-m_{5}(\mathbf{p c s})$ & $s_{1}$ & $s_{1,1}$ & $s_{1,-1}$ & $t_{1}$ & $t_{1}$ & $t_{1,-1}$ \\
\hline \begin{tabular}{|l|}
$S^{2}$ \\
{$[200,400]$} \\
{$[300,500]$}
\end{tabular} & $\begin{array}{l}5.52 \pm 0.08 \\
4.18 \pm 0.94 \\
1.90 \pm 2.23\end{array}$ & $\begin{array}{l}1.52 \pm 0.01 \\
1.82 \pm 0.20 \\
2.24 \pm 0.50\end{array}$ & $\left|\begin{array}{l}-1.04 \pm 0.01 \\
-1.10 \pm 0.04 \\
-1.18 \pm 0.15\end{array}\right|$ & $\begin{array}{l}7.89 \pm 0.01 \\
7.05 \pm 0.50 \\
7.05 \pm 0.45\end{array}$ & $\begin{array}{l}2.88 \pm 0.02 \\
2.48 \pm 0.30 \\
2.80 \pm 0.04\end{array}$ & $\mid \begin{array}{l}0.97 \pm 0.01 \\
1.62 \pm 0.40 \\
0.07 \pm 0.75\end{array}$ \\
\hline $\mid N o-K M-m_{5}$ (pcs) & $s_{1,0}$ & $s_{1,1}$ & $s_{1,-1}$ & ${ }^{{ }_{1,0}}$ & $t_{1,1}$ & $t_{1,-1}$ \\
\hline \begin{tabular}{|l|}
$S^{2}$ \\
{$[200,400]$} \\
{$[300,500]$}
\end{tabular} & \begin{tabular}{|c|}
$10.24 \pm 0.01$ \\
$8.07 \pm 0.14$ \\
$7.92 \pm 0.07$
\end{tabular} & $\left|\begin{array}{l}2.62 \pm 0.10 \\
4.12 \pm 0.38 \\
3.48 \pm 0.10\end{array}\right|$ & $\begin{array}{l}1.14 \pm 0.02 \\
2.04 \pm 0.60 \\
2.43 \pm 0.43\end{array}$ & $\begin{array}{l}2.05 \pm 0.01 \\
3.40 \pm 0.90 \\
4.09 \pm 0.70\end{array}$ & $\begin{array}{l}-0.08 \pm 0.02 \\
-1.43 \pm 0.70 \\
-2.72 \pm 0.80\end{array}$ & $\mid \begin{array}{l}4.56 \pm 0.02 \\
5.84 \pm 0.20 \\
5.16 \pm 0.16\end{array}$ \\
\hline
\end{tabular}

with magnitudes in the $\mathrm{m} 5$ and $\mathrm{m} 6$ groups (already candidates per se, because of the behavior observed in the previous study considering magnitudes) and both KM and noKM. The obtained results are given in Table 2. The results of Marco et al. (2017) for a slice around 400 pcs are similar to the expected results for the $m_{6}$ or $m_{5}+m_{6}$ set from table 1 . The difference in the $t_{1,-1}$ coefficient is due to the bias in $\delta$ in that slice. Further considerations and interpretations of the coefficients will be provided in a later paper, now in preparation.

\section{Acknowledgements}

This work was supported by a grant UJI-B2016-18.

\section{References}

Marco, F. J., Martínez, M. J. \& López J. A. 2017, Astrometry and Astrophysics in the Gaia Sky, In Press. 